\title{
Cardiac-specific disruption of the c-raf-1 gene induces cardiac dysfunction and apoptosis
}

\author{
Osamu Yamaguchi, ${ }^{1}$ Tetsuya Watanabe, ${ }^{1}$ Kazuhiko Nishida, ${ }^{1,2}$ Kazunori Kashiwase, ${ }^{1}$ \\ Yoshiharu Higuchi, ${ }^{1}$ Toshihiro Takeda, ${ }^{1}$ Shungo Hikoso, ${ }^{1}$ Shinichi Hirotani, ${ }^{1}$ Michio Asahi, ${ }^{1}$ \\ Masayuki Taniike, ${ }^{1}$ Atsuko Nakai, ${ }^{1}$ Ikuko Tsujimoto, ${ }^{3}$ Yasushi Matsumura, ${ }^{4}$ Jun-ichi Miyazaki, ${ }^{5}$ \\ Kenneth R. Chien, ${ }^{6}$ Atsushi Matsuzawa,7 Chiharu Sadamitsu,7 Hidenori Ichijo,7 \\ Manuela Baccarini, ${ }^{8}$ Masatsugu Hori, ${ }^{1}$ and Kinya Otsu ${ }^{1}$
}

\begin{abstract}
1Department of Internal Medicine and Therapeutics, Osaka University Graduate School of Medicine, Suita, Osaka, Japan. ${ }^{2}$ Department of Dental Anesthesiology, ${ }^{3}$ First Department of Oral and Maxillofacial Surgery, Osaka University Graduate School of Dentistry, Suita, Osaka, Japan.

4Department of Medical Information Science, ${ }^{5}$ Division of Stem Cell Regulation Research, Osaka University Graduate School of Medicine, Suita, Osaka, Japan.

${ }^{6}$ Department of Medicine, School of Medicine, University of California, San Diego, La Jolla, California, USA. 'Laboratory of Cell Signaling and Core Research for Evolutional Science and Technology, Graduate School of Pharmaceutical Sciences, University of Tokyo, Tokyo, Japan. ${ }^{8}$ Department of Microbiology and Immunology, Vienna Biocenter, Institute of Microbiology and Genetics, Vienna, Austria.
\end{abstract}

\begin{abstract}
The Raf/MEK/extracellular signal-regulated kinase (ERK) signaling pathway regulates diverse cellular processes such as proliferation, differentiation, and apoptosis and is implicated as an important contributor to the pathogenesis of cardiac hypertrophy and heart failure. To examine the in vivo role of Raf- 1 in the heart, we generated cardiac muscle-specific Raf-1-knockout (Raf CKO) mice with Cre-loxP-mediated recombination. The mice demonstrated left ventricular systolic dysfunction and heart dilatation without cardiac hypertrophy or lethality. The Raf CKO mice showed a significant increase in the number of apoptotic cardiomyocytes. The expression level and activation of MEK1/2 or ERK showed no difference, but the kinase activity of apoptosis signal-regulating kinase 1 (ASK1), JNK, or $\mathrm{p} 38$ increased significantly compared with that in controls. The ablation of ASK1 rescued heart dysfunction and dilatation as well as cardiac fibrosis. These results indicate that Raf-1 promotes cardiomyocyte survival through a MEK/ERK-independent mechanism.
\end{abstract}

\section{Introduction}

Heart failure, one of the leading causes of both morbidity and mortality in developed countries, is the convergent phenotype of various diseases that cause loss and dysfunction of cardiomyocytes. Series of parallel and convergent signaling pathways are activated that lead to the common phenotype of heart failure, but molecular mechanisms underlying the pathogenesis of heart failure await clarification. Recent studies have indicated that apoptosis may be an important feature of heart failure (1).

Raf is a family of 3 serine/threonine-specific kinases (A-Raf, B-Raf, and Raf-1) ubiquitously expressed throughout embryonic development. The principal function of the Raf protein kinases appears to be participation in the highly conserved Ras/Raf/MEK/ extracellular signal-regulated kinase (ERK) intracellular signaling pathway, which has been implicated in the transduction of signals directing cell proliferation and differentiation. In addition, Raf-1 activation of the MEK/ERK pathway has been associated with inhibition of apoptosis, leading to cell survival $(2,3)$.

To determine the in vivo role of Raf- 1 , Raf- 1 knockout mice were generated $(4,5)$. The embryos of these mice are growth retarded and die progressively around midgestation with defects in the placenta and in the liver due to apoptosis (4). The phenotype observed

Nonstandard abbreviations used: ASK1, apoptosis signal-regulating kinase 1; CTL, control littermate of $c$-raf-1 $1^{\text {flox/flox }}: \alpha-M H C C r e(-)$ mice; ERK, extracellular signal-regulated kinase; FS, fractional shortening; LV, left ventricle/ventricular; $\alpha$-MHCCre, transgenic mice expressing Cre recombinase under the control of the $\alpha$-myosin heavy chain promoter; MKK, mitogen-activated protein kinase kinase; Raf CKO, cardiac muscle-specific Raf-1 knockout; RV, right ventricle.

Conflict of interest: The authors have declared that no conflict of interest exists.

Citation for this article: J. Clin. Invest. 114:937-943 (2004)

doi:10.1172/JCI200420317. appears to be the result of a lack of activation of Raf-1-specific effectors distinct from the ERK pathway (4-6). At present, the molecular mechanisms underlying the MEK/ERK-independent prosurvival function of Raf-1 are poorly understood. A recent study has demonstrated that Raf-1 physically interacts with the proapoptotic, stressactivated mitogen-activated protein kinase kinase kinase, apoptosis signal-regulating kinase 1 (ASK1) (7), which is a key element in the mechanism of stress- and cytokine-induced apoptosis $(8,9)$. A variety of stress-related stimuli activate ASK1, including TNF- $\alpha$, reactive oxygen species, and Fas. ASK1 phosphorylates and activates mitogen-activated protein kinase kinase 4/7 (MKK4/7) and MKK3/6, which in turn activate JNK and p38 MAPK, respectively.

In the heart, the MEK/ERK pathway has been implicated as the signaling cascade leading to hypertrophy of cardiomyocytes (10-12), although a number of additional studies have disputed the importance of the pathway in the regulation of cardiac hypertrophy (13-15). A recent study of MEK1 transgenic mice has suggested that the MEK/ERK signaling pathway stimulates a hypertrophic response associated with resistance to apoptosis (16). However, the in vivo role of Raf-1 in the heart has not been identified yet. In this study, we generated cardiac-specific Raf-1 knockout (Raf CKO) mice to clarify the role of Raf-1 in the heart. These mice showed left ventricular (LV) dysfunction and heart dilatation with an increased number of apoptotic cells. These phenotypes were rescued by inactivation of ASK1. To the best of our knowledge, this is the first study demonstrating that Raf-1 plays a role at the organ level.

\section{Results}

Generation of cardiac muscle-specific Raf-1 knockout mice. To obtain a cardiac muscle-specific knockout of the c-raf-1 gene, we used Cre- 


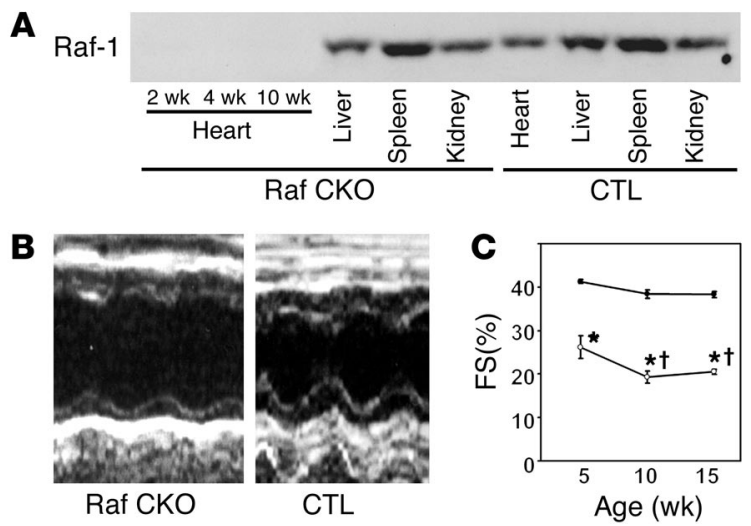

Figure 1

Characterization of Raf CKO mice. (A) The expression level of Raf-1 in indicated tissues was examined with the anti-Raf- 1 antibody. Tissue homogenates were extracted from CTLs and Raf CKO mice. (B) Transthoracic M-mode echocardiographic tracings in a Raf CKO mouse (left) and a CTL (right) at 10 weeks of age. (C) Changes in fractional shortening (FS) at 5, 10, and 15 weeks in Raf CKO mice (open circles) and CTLs (filled circles). ${ }^{*} P<0.05$ versus controls at the same age. t $P<0.05$ versus Raf CKO at 5 weeks.

loxP strategies, which employ c-raf-1 floxed-allele mice (6) and transgenic mice expressing the Cre recombinase under the control of the $\alpha$-myosin heavy chain promoter ( $\alpha$-MHCCre mice) (17). This floxed $c$-raf- 1 allele contains 2 loxP sites, which have been inserted at $3^{\prime}$ and $5^{\prime}$ of exon 3 of the $c$-raf-1 gene (designated "c-raf-1flox"). Mice homozygous to the c-raf-1 $1^{\text {flox }}$ allele mice appeared to be phenotypically indistinguishable from wild-type animals, and the protein levels of Raf- 1 were similar to those in wild-type mice (6). To achieve conditional inactivation of the c-raf-1 gene in cardiac muscle, $c$-raf-1 flox/flox mice were crossed with $\alpha$-MHCCre mice. To demonstrate tissue-specific deficiency of Raf-1, we used Western blot analysis to examine the protein levels of Raf- 1 in various tissues extracted from $c$-raf-1 flox/flox: $\alpha-M H C C r e(+)$ mice, which harbor Raf CKO. Immunoblotting indicated a marked reduction in the Raf-1 protein exclusively in the heart of Raf CKO mice with no detectable reductions in other tissues such as liver, spleen, and kidney (Figure 1A). In addition, there was almost no difference in cardiac Raf-1

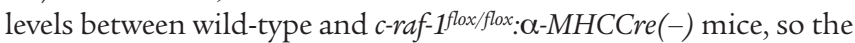
latter were used as controls (data not shown).

Conditional depletion of Raf-1 developed heart dysfunction. Expected mendelian ratios of $c$-raf-1 flox/flox: $\alpha-M H C C r e(-)$ and $c$-raf-1 flox/flox: $\alpha-\operatorname{MHCCre}(+)(n=121$ and 114, respectively) were detected between

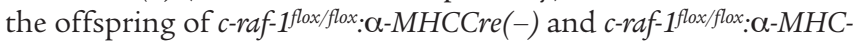
$\mathrm{Cre}(+)$, indicating no significant embryonic lethality. The Raf CKO mice were born normally and were externally indistinguishable from control littermates of $c$-raf-1flox/flox: $\alpha-M H C C r e(-)$ mice (CTLs). The mice developed to adulthood, were fertile, and exhibited a normal lifespan up to 12 months of age. Thus, the $c$-raf- 1 gene is not essential for mouse heart development.

To investigate whether the cardiac-specific knockout of Raf-1 would affect in vivo cardiac function, cardiac performance was analyzed by means of echocardiography at 10 weeks of age (Table 1; Figure 1B). Cardiac contractility was significantly depressed in Raf CKO mice compared with that in CTLs as assessed by fractional shortening (FS). The LV end-diastolic and end-systolic diameters significantly increased in Raf CKO mice compared with
CTLs. Although there were no significant differences in septal wall thickness between Raf CKO mice and CTLs, posterior wall thickness significantly decreased in Raf CKO mice. Catheterization of the $\mathrm{LV}$ showed a marked reduction in the maximum or minimum first derivative of $\mathrm{LV}$ pressure in Raf CKO mice, thus demonstrating a reduction in myocardial contractility and relaxation (Table 2 ). There were no significant differences in heart rate, systolic and diastolic pressure, or LV end-diastolic pressure. The mice were followed up at 5, 10, and 15 weeks of age (Figure 1C). In Raf CKO mice, FS was significantly decreased at 10 weeks compared with that at 5 weeks but remained unchanged thereafter.

The Raf CKO mice developed enlarged hearts (Figure 2A) but displayed no differences in heart weight or the average ratio of heart weight to tibia length or to body weight (Table 2). In addition, there were no significant differences in the weight of the LV, RV, atrium, lung, or liver. Histological examination of the heart demonstrated LV enlargement in Raf CKO hearts (Figure 2B), while also demonstrating that the cross-sectional area of cardiomyocytes exhibited no significant differences between Raf CKO mice and CTLs $\left(174.2 \pm 6.8 \mu \mathrm{m}^{2}\right.$ for Raf CKO mice and $184.6 \pm 7.7 \mu \mathrm{m}^{2}$ for CTLs) (Figure 2C). These findings are consistent with LV pump dysfunction without ventricular pump failure. In addition, either endogenous Raf-1 does not play a role in the regulation of developmental cardiomyocyte hypertrophic growth, or its function in the growth process is offset by that of other MEK activators. Masson trichrome staining revealed significant fibrosis in intermuscular areas in Raf CKO mice compared with those in CTLs (Figure 2D).

Increased apoptosis in hearts with Raf-1 conditional depletion. Raf-1 activation of the MEK/ERK pathway is associated with inhibition of apoptosis, leading to cell survival $(2,3,18)$. Therefore, it can be hypothesized that the cardiac dysfunction observed in Raf CKO mice was associated with an increase in myocyte apoptosis. TUNEL assay revealed a significant increase in apoptotic cells in Raf CKO hearts at 3, 4, and 5 weeks of age but did not at 2 weeks and after 6 weeks of age (Figure 3A). The appearance of apoptosis was further confirmed by morphological examination, which demonstrated condensed chromatin and fragmented nuclei in TUNEL-positive cells; these are characteristics of apoptosis. The TUNEL-positive cells were identified as cardiac myocytes by anti- $\alpha$-sarcomeric actin staining (Figure 3B).

Analysis of apoptosis-related proteins showed an increased level of Bax in Raf CKO mice compared with CTLs. No significant difference was observed in the level of Bcl-2 (Figure 3C). The ratio of

\section{Table 1}

Echocardiographic measurements of the knockout mice at 10 weeks of age

$\begin{array}{lcc} & \text { Raf CKO }(\boldsymbol{n}=\mathbf{1 2}) & \text { CTLs }(\boldsymbol{n}=\mathbf{1 2}) \\ \text { LVDd }(\mathrm{mm}) & 4.79 \pm 0.14^{\mathrm{A}} & 4.16 \pm 0.08 \\ \text { LVDs }(\mathrm{mm}) & 3.89 \pm 0.16^{\mathrm{A}} & 2.57 \pm 0.08 \\ \text { FS }(\%) & 19.3 \pm 1.4^{\mathrm{A}} & 38.4 \pm 1.0 \\ \text { IVSd }(\mathrm{mm}) & 0.76 \pm 0.04 & 0.82 \pm 0.04 \\ \text { LVPWd }(\mathrm{mm}) & 0.49 \pm 0.02^{\mathrm{A}} & 0.58 \pm 0.04 \\ \text { Heart rate (beats/min) } & 473 \pm 16 & 491 \pm 15\end{array}$

LVDd, diastolic left ventricle internal dimension; LVDs, systolic left ventricle internal dimension; IVSd, diastolic interventricle septal wall thickness; LVPWd, diastolic left ventricle posterior wall thickness. Data are expressed as mean \pm SEM. ${ }^{A} P<0.05$ versus $C T L s$. 


\section{Table 2}

Physiological base-line parameters of Raf CKO mice at 10 weeks of age

$\begin{array}{lcc} & \text { Raf CKO }(\boldsymbol{n}=\mathbf{9}) & \text { CTLs }(\boldsymbol{n}=\mathbf{7}) \\ \text { Body weight }(\mathrm{g}) & 27.1 \pm 1.0 & 28.8 \pm 1.2 \\ \text { Heart weight }(\mathrm{mg}) & 130 \pm 4 & 132 \pm 7 \\ \text { LV weight }(\mathrm{mg}) & 89.4 \pm 3.6 & 89.6 \pm 4.2 \\ \text { RV weight }(\mathrm{mg}) & 24.1 \pm 1.5 & 26.1 \pm 1.6 \\ \text { Atrium weight }(\mathrm{mg}) & 12.6 \pm 0.6 & 13.2 \pm 1.2 \\ \text { Lung weight }(\mathrm{mg}) & 141 \pm 1 & 149 \pm 5 \\ \text { Tibia length }(\mathrm{mm}) & 17.7 \pm 0.2 & 17.9 \pm 0.1 \\ \text { Heart rate }(\text { beats/min) } & 391 \pm 33 & 427 \pm 24 \\ \text { Systolic blood pressure }(\mathrm{mmHg}) & 84.6 \pm 4.2 & 86.8 \pm 5.1 \\ \text { LV systolic pressure (mmHg) } & 82.4 \pm 2.6 & 86.0 \pm 4.1 \\ \text { LVEDP }(\mathrm{mmHg}) & 1.3 \pm 0.3 & 1.1 \pm 0.4 \\ \text { LV dp/dt max (mmHg/s) } & 6,733 \pm 411^{\mathrm{A}} & 9,657 \pm 835 \\ \text { LV dp/dt min }(\mathrm{mmHg} / \mathrm{s}) & -4,344 \pm 287 \mathrm{~A} & -5,957 \pm 796\end{array}$

LVEDP, left ventricular end-diastolic pressure. LV dp/dt max and LV dp/dt min are the maximum rates of pressure development during contraction and relaxation, respectively. Data are expressed as mean \pm SEM. ${ }^{A} P<0.05$ versus $C T L s$.

$\mathrm{Bcl}-2$ protein to Bax protein determines survival or death after an apoptotic stimulus (19). The Bax/Bcl-2 ratio in Raf CKO mice was significantly larger than that in CTLs (Figure 3C).

MAPK activation in hearts with Raf-1 depletion. To test the activation profile of MEK1/2 or ERK in Raf CKO hearts, the protein level of phosphorylated MEK1/2 or ERK was measured after 3 minutes of intra-arterial infusion of endothelin-1 (Figure 4A). A similar level of MEK1/2 or ERK phosphorylation was observed in Raf CKO and CTL hearts in response to endothelin-1. This indicates that the MEK/ERK pathway was not impaired in Raf CKO hearts. To examine whether the MEK/ERK pathway is involved in cardiac dysfunction observed in Raf CKO mice, we assessed activation of MEK1/2 or ERK in the hearts. There were no differences in the basal protein levels of ERK or MEK1/2 in Raf CKO and CTL hearts, nor in the level of phosphorylated ERK or MEK1/2 (Figure 4, B and C). The phosphorylation level of p38 significantly increased at 3 weeks of age (Figure 4C). The phosphorylation level of JNK at 3 and 4 weeks of age was significantly enhanced $(2.49 \pm 0.59$-fold increase at 4 weeks) in Raf CKO mice compared with that in CTLs (Figure 4C). We observed no differences in Akt activation in Raf CKO mice compared with that in CTLs. This implies that Raf-1 activity is not required for ERK activation, presumably because other Raf isoforms or MEK activators compensate for the loss of Raf-1. Raf- 1 appears to promote cell survival in the heart through a MEK/ERK-independent mechanism. In addition, these results imply a role of JNK or p38, but not ERK, in cardiac dysfunction in Raf CKO mice.

ASK1 ablation rescues abnormalities in mice with Raf-1 conditional depletion. A recent in vitro study suggests that Raf-1 promotes

\section{Figure 2}

Cardiac histological analysis of Raf CKO mice. (A) Photographs of hearts removed from CTLs (right) and Raf CKO (left) mice at 10 weeks of age. (B) Macroscopic H\&E-stained histological section of hearts from CTLs (right) and Raf CKO (left) mice at 10 weeks of age. (C) Microscopic H\&Estained sections of hearts from CTLs (right) and Raf CKO (left) mice. (D) Microscopic Masson trichrome-stained section of hearts from CTLs (right) and Raf CKO (left) mice. Scale bar: 2 mm (B), $100 \mu \mathrm{m}$ (C and D). cell survival by antagonizing ASK1 (7). The ASK1/JNK signaling pathway has been reported to be a crucial element of neuronal or cardiac apoptosis $(20,21)$. We first used the immunoprecipitation kinase assay to compare the activity of the endogenous ASK1 proteins in Raf CKO and CTL hearts. The in vitro kinase assay clearly showed an increase in the kinase activity of ASK1 in Raf CKO hearts compared with controls at 4 weeks of age (Figure 5), but no significant difference at 2 or 10 weeks of age. To determine whether the ASK1 activation in Raf CKO hearts results in cardiac dysfunction and dilatation, we generated the Raf-1/ASK1 double-knockout [c-raf-1 $\left.1^{\text {flox/flox }}: \alpha-\operatorname{MHCCre}(+): A S K^{-/}\right]$line by mating Raf CKO with conventional ASK1 knockout mice. As we previously reported, ASK1 knockout mice were born at the expected mendelian frequency, were indistinguishable in appearance from wild-type littermates (9), and displayed no differences in either physiological parameters or cardiac performance as analyzed by means of echocardiography and hemodynamic measurement (21). The Raf-1/ASK1 double-knockout mice were born normally and were fertile. The increase in heart size appeared to be reduced in double-knockout mice compared with that in c-raf-1 $1^{\text {flox/flox: }} \alpha-\mathrm{MHC}$ $\mathrm{Cre}(+): \mathrm{ASK}^{+/+}$mice (RafCKO:ASK ${ }^{++}$) (Figure $\left.6 \mathrm{~A}\right)$. The protein level of Raf- 1 in the double-knockout mice was significantly reduced, as in Raf CKO:ASK ${ }^{+/+}$mice (Figure 6B). LV end-diastolic diameter was significantly reduced and FS increased in the Raf-1/ASK1 double-knockout mice compared with Raf CKO:ASK ${ }^{+/+}$mice (Fig-

A
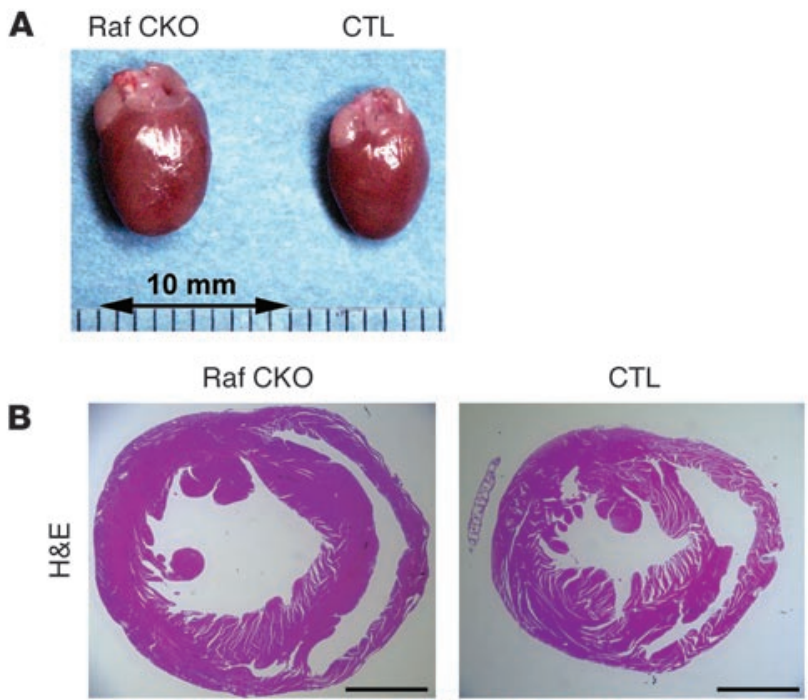

C
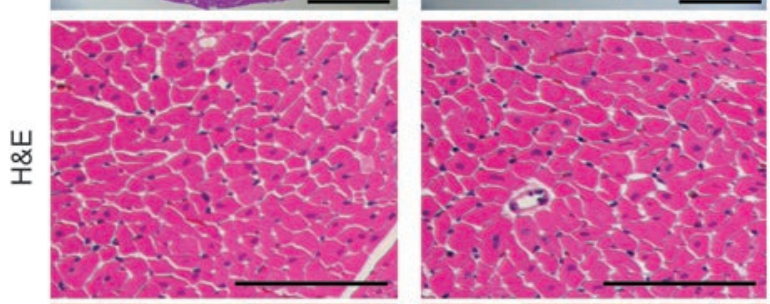

D

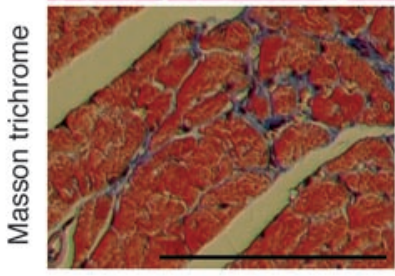



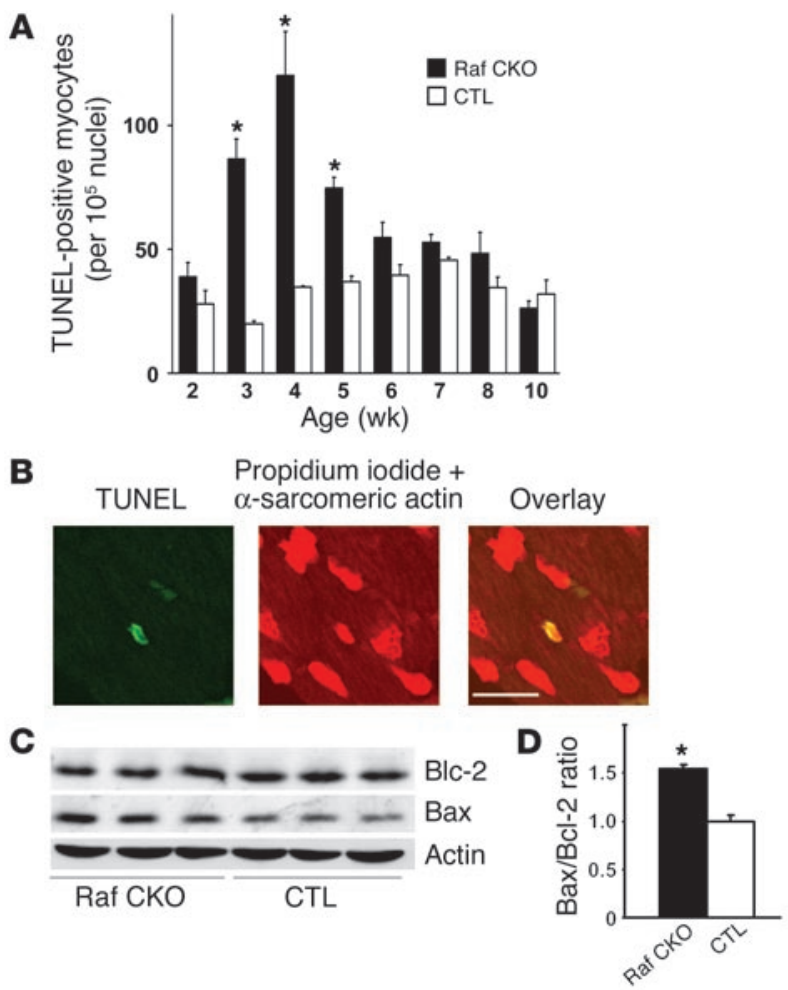

Figure 3

Apoptosis in Raf CKO hearts. (A) Number of TUNEL-positive cells in Raf CKO hearts (black bars) at 2, 3, 4, 5, 6, 7, 8, and 10 weeks of age compared with CTL hearts (white bars) $(n=3)$. ${ }^{*} P<0.05$ versus CTLs at the same age. (B) Confocal analysis of Raf CKO ventricular myocardium. Triple staining (propidium iodide, TUNEL, and anti$\alpha$-sarcomeric actin antibody) was performed. Staining for propidium iodide and anti- $\alpha$-sarcomeric actin antibody is shown in red, and that for TUNEL in green. In the overlay image, a nucleus stained by both TUNEL and propidium iodide is shown in yellow. Scale bar: $20 \mu \mathrm{m}$. (C) Immunoblot analysis of the apoptosis-related proteins in Raf CKO hearts. The expression levels of $\mathrm{Bcl}-2$, Bax, and actin as a control in the indicated tissues were examined with their respective antibodies. (D) The Bax/Bcl-2 ratio is shown $(n=3)$.

ure $6, \mathrm{C}$ and $\mathrm{D})$. The value of $\mathrm{LV}$ end-diastolic diameter or FS in Raf-1/ASK1 double-knockout mice was similar to that in CTLs: $A S K^{+/+}$and CTL: $A S K^{-/-}$mice. The double knockout rescued the pathology in Raf CKO hearts (Figure 6E). The double-knockout hearts exhibited less fibrosis compared with Raf CKO:ASK ${ }^{+/+}$. The phosphorylation levels of JNK and p38 were reduced in the Raf-1/ ASK1 double-knockout mice compared with $\mathrm{Raf} \mathrm{CKO} / \mathrm{ASK}^{+/+}$ littermates (Figure 6F). The number of TUNEL-positive cardiomyocytes significantly decreased in the double-knockout mice at 4 weeks of age, but not 10 weeks (Figure 6G). Thus, ablation of ASK1 in cardiomyocytes rescued functional, structural, and histological abnormalities observed in Raf CKO mice. This indicates that ASK1 activation in Raf CKO hearts might be involved in the pathogenesis of cardiac dysfunction.

\section{Discussion}

In order to better understand the function of Raf- 1 in mature cardiac muscle, we used the Cre-loxP system to specifically inactivate the $c$-raf-1 gene in cardiac muscle. Our data indicate that the c-raf-1 gene is not essential for mouse heart development or the regulation of developmental cardiomyocyte hypertrophic growth. Our data, however, do not exclude the role of the MEK/ERK pathway in cardiac development or hypertrophy, since MEK1/2 and ERK can be activated in Raf CKO hearts.

Raf CKO mice exhibited cardiac dysfunction and heart dilatation at 5 weeks of age. Raf CKO hearts demonstrated a heightened level of apoptosis at 3, 4, and 5 weeks but not at 2 weeks and after 6 weeks of age, even though cardiac contractility progressively decreased during the 10 weeks. These findings suggest that apoptosis is one of the early causes of cardiac dysfunction rather than a terminal event associated with the end stage. Mice are undergoing marked postnatal growth and hypertrophic stimulation during the first month after birth. We assume that such stress might transiently activate stress-activated signaling molecules such as ASK1 in Raf $\mathrm{CKO}$ mice and induce cardiomyocyte apoptosis. To ensure that the resultant anatomical and functional alterations were not in part due to positional effects of the Cre gene, we crossed c-raf-1 flox/flox mice with mice expressing the Cre recombinase under the control of the myosin light chain $2 \mathrm{v}$ (MLC2v) promoter (22). We observed similar phenotypes in the c-raf-1 flox/flox:MLC2v-Cre ${ }^{+/-}$mice.

Accumulating evidence suggests that apoptosis may be an important mode of cell death during heart failure in both humans and animal models (1). However, the extent to which apoptosis plays a critical role in the pathogenesis of heart failure has remained an open question. Our study demonstrates that induction of apoptosis leads to the development and progression of cardiac dysfunction. A number of biochemical studies have indicated a role for Raf-1 in apoptosis $(2,3)$. Recent knockout studies of Raf-1 demonstrated that $c$-raf- $1^{-/-}$mice die in embryogenesis and show vascular defects in the yolk sac and placenta as well as increased apoptosis in embryonic tissues $(4,5)$. These findings suggest that Raf-1 plays a key role in preventing apoptosis, which is in agreement with the findings of our study of the heart. However, it is possible that ablation of Raf- 1 affected gene expression of calcium-handling proteins, other ion-handling proteins, and contractile proteins, which regulate cardiac contractility. We observed no changes in protein expression of calcium-handling proteins such as ryanodine receptor, SERCA, phospholamban, and calsequestrin (data not shown). As a limitation of knockout study, we could not exclude the possibility that ablation of one gene leads to alteration of the expression profile of unrelated or related genes. In this study, Raf CKO hearts did not develop compensatory hypertrophy despite cardiac dysfunction and dilatation, which suggests that Raf- 1 is involved in compensatory cardiomyocyte growth through a MEK/ERK-independent pathway. This could be another important mechanism of cardiac dysfunction in Raf CKO mice. Further study will be necessary to elucidate a role of Raf- 1 in compensatory hypertrophy in response to increased hemodynamic stress.

Raf- 1 activation of the MEK/ERK pathway has been associated with inhibition of apoptosis $(2,3)$. However, studies using conventional $c-r a f-1^{-/-}$mice suggest that effectors other than the MEK/ERK cascade must mediate the antiapoptotic function of Raf-1 $(4,5)$. Our results indicate that Raf-1 promotes cell survival in the heart, as in other tissues, through a MEK/ERK-independent mechanism. In this study, we detected a transient increase in ASK1 activity in Raf CKO hearts. Furthermore, ablation of ASK1 resulted in the rescue of the functional, structural, and histological phenotypes observed in Raf CKO mice. These suggested that ASK1 might play an important role in the apoptosis-induced abnormalities observed in Raf-1-knockout 
A

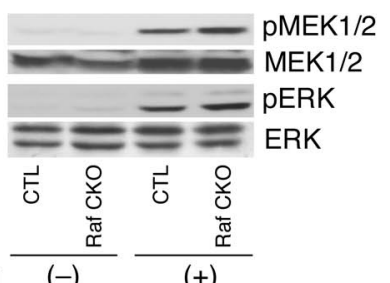

C

C $_{\text {Age }}$

$(+)$

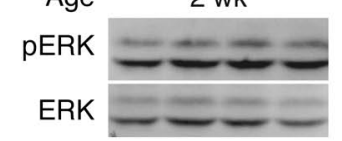

pJNK

JNK

p-p38

p38

pAkt

Akt

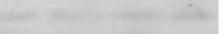

-
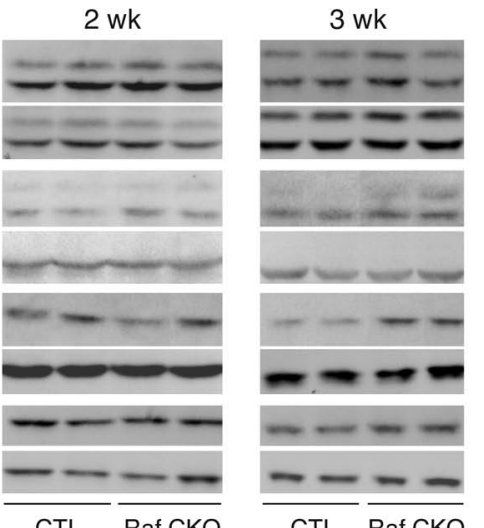

CTL Raf CKO

$7 \mathrm{wk}$
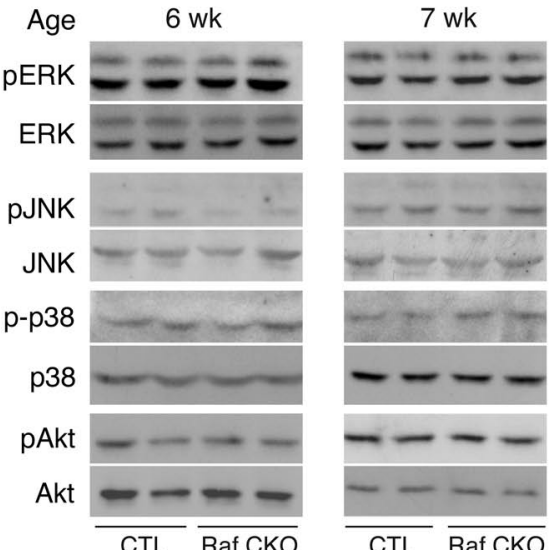

$\overline{\mathrm{CTL}} \overline{\text { Raf CKO }}$

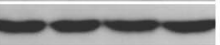

B

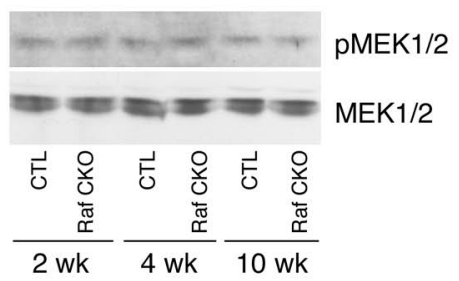

4 wk
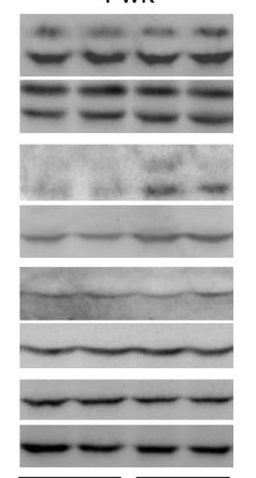

CTL $\overline{\text { Raf CKO }}$

$8 \mathrm{wk}$
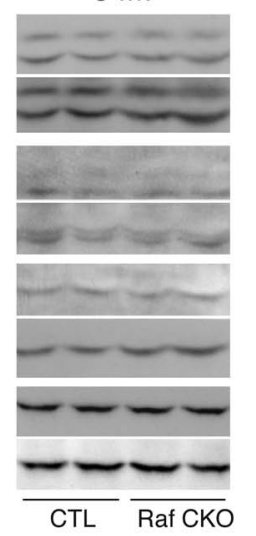

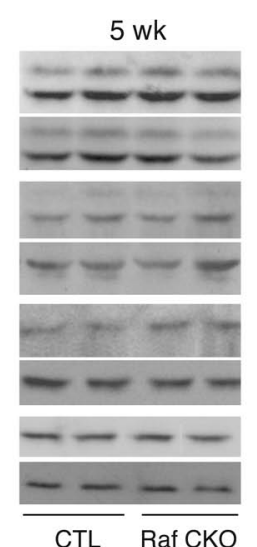

CTL Raf CKO 10 wk

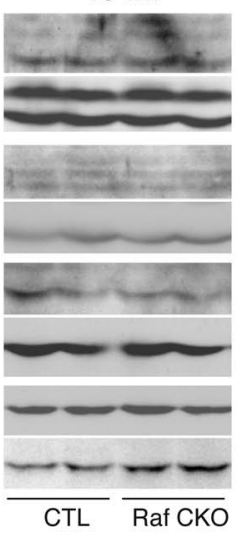

\section{Figure 4}

Western blot analysis of Raf CKO mice. The phosphorylation levels of MEK1/2, ERK, p38, JNK, and Akt were assessed by immunoblotting with antiphosphospecific antibody. The filters were reprobed with anti-nonphosphospecific antibody. (A) Western blot analysis of MEK1/2 and ERK phosphorylation in the hearts of Raf CKO mice and CTLs after 3 minutes of perfusion with endothelin-1 (ET-1) or PBS stimulation. (B) Western blot analysis of MEK1/2 phosphorylation in the hearts of Raf CKO mice and CTLs at 2, 4, and 10 weeks of age. (C) Western blot analysis of ERK, p38, JNK, and Akt phosphorylation in the hearts of Raf CKO mice and CTLs at 2, 3, 4, 5, 6, 7, 8, and 10 weeks of age.

We detected a transient activation of JNK and p38 in Raf CKO mice. We observed no activation of JNK and p38 in the Raf-1/ASK1 doubleknockout mice. Knockout studies indicate the role of the JNK pathway in proapoptotic signaling (24). The ASK1/JNK signaling pathway has been reported to be a crucial element of cardiac (21) and neuronal apoptosis (20). Thus, we can hypothesize that the activation of the ASK1/ JNK signaling pathway might mediate apoptosis in Raf CKO hearts. In addition to JNK activation, it is also possible that p38 activation might be involved in the apoptotic pathway.

The molecular target for activated ASK1 to induce apoptosis remains to be elucidated. It has been reported that overexpression of a dominant negative mutant of ASK induced cytochrome $c$ release from mitochondria and activation of caspase- 9 and caspase- 3 , but not of caspase-8, in nonmuscle cells (25). This indicates that mitochondria-initiated activation of caspases might be a main mechanism operating in the execution of ASK1-induced apoptosis. The ratio of Bcl-2 protein to Bax protein deterhearts. Overexpression of wild-type or constitutively active ASK1 induces apoptosis in various cells (23), while oxidative stress and TNF-induced apoptosis are suppressed in $A S K 1^{-/}$cells $(6,9)$. We have previously shown that expression of a constitutively active mutant of ASK1 in cardiomyocytes results in apoptosis, while isolated $\mathrm{ASK}^{-/-}$ cardiomyocytes are resistant to $\mathrm{H}_{2} \mathrm{O}_{2}$ (21). The ablation of ASK1 in mice prevents heart dilatation and cardiac dysfunction following pressure overload or myocardial infarction, possibly by inhibiting the ASK/JNK signaling pathway (21). The prevention of LV remodeling was accompanied by a reduction in the appearance of apoptotic cardiomyocytes in $\mathrm{ASK}^{-1-}$ hearts. Thus, the activation of ASK1 in Raf CKO mice appears to be a cause of cardiac dysfunction and heart dilatation. However, it is still possible that, in mouse hearts in which both the ASK1 and the Raf-1 genes have been ablated, the beneficial effect of ASK1 ablation on LV remodeling independently cancels the detrimental effect of Raf- 1 ablation. Alternative pathways aside from ASK1, which could contribute to alteration of cell survival, might mediate the phenotypes observed in Raf CKO mice. As a candidate, we examined Akt activation in RafCKO mice. We observed no difference in Akt activation between Raf CKO mice and CTLs. mines survival or death after an apoptotic stimulus (19). In our study, we observed a decrease in the ratio of Bcl-2 to Bax in Raf CKO hearts, which promotes cell death. However, the mechanism underlying this increase in Bax protein level in response to ASK1 activation in Raf CKO mice remains to be clarified.

It is currently unclear precisely how the Raf- 1 protein regulates ASK1 activation and whether ASK1 activation is a direct or an indirect consequence of Raf- 1 ablation. The recent demonstration of interaction between Raf-1 and ASK1 indicates that Raf-1 may pro-

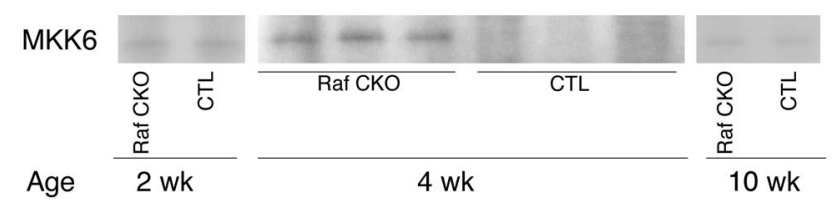

Figure 5

ASK1 activity in Raf CKO mice. ASK1 activity was measured by immune complex assay, using His-MKK6 as a substrate. Panels represent ASK1 activation at 2, 4, and 10 weeks of age. 
mote cell survival through its protein-protein interactions, which are not related to Raf-1 kinase activity (7). It is possible to hypothesize that Raf-1 promotes cardiomyocyte survival by antagonizing ASK1. Deletion of the N-terminal domain of ASK1 caused the constitutive activation of kinase activity and induction of apoptosis. Raf-1 has been shown to bind to the N-terminal domain of ASK1 (7). Thus, Raf-1 might promote an inactive conformation of ASK1 through the interaction of Raf- 1 with the $\mathrm{N}$-terminal domain of ASK1. In addition, the Fas death receptor-associated protein Daxx has been found to activate ASK1 through interaction with the N-terminal portion of ASK1 and lead to apoptosis (26). It is, therefore, also possible that Raf-1 interferes with the binding of ASK1 with Daxx; another possibility is that the antagonizing function of Raf-1 against ASK1 is mediated through Akt activation. It has been reported that Raf-1 is associated with Akt (27), which phosphorylates and negatively regulates ASK1 (28). These findings indicate that Raf-1 may recruit Akt to phosphorylate ASK1, resulting in reduced ASK1 kinase activity. However, we could not detect any change of Akt activation in Raf CKO mice. It has been reported that $\mathrm{H}$-Ras interacts with ASK1 to cause the inhibition of both ASK1 activity and ASK1-induced apoptosis (29). We observed no significant difference in the protein expression level of $\mathrm{H}$-Ras between Raf CKO and CTL hearts (data not shown); this excludes the possibility that altered expression levels of H-Ras in Raf CKO hearts could contribute to enhancement of ASK1 activity.

To summarize, RafCKO mice exhibited cardiac dysfunction and dilatation with increased apoptosis. Activation of the ASK1 signaling pathway seems to play an important role in apoptosis in Raf-1-knockout hearts.

\section{Methods}

Generation of the Raf CKO mice. The construction of $c$-raf-1 $1^{\text {flox/flox }}$ and ASK1depleted mice has been previously described in detail $(6,9)$. The transgenic mice expressing the Cre recombinase under the control of $\alpha$-myosin heavy chain promoter ( $\alpha$-MHCCre mice) in C57BL/6J background were generated as previously reported (17). To confirm the cardiac-specific expression of the Cre recombinase in the $\alpha$-MHCCre mice, they were mated with a transgenic mouse line carrying a reporter gene construct, which directs expression of the Escherichia coli lac $Z$ gene following Cre-mediated excision

\section{Figure 6}

Prevention of cardiac dysfunction in Raf-1/ASK1 double-knockout mice. (A) Photographs of hearts removed from $c$-raf-1 floxflox: $\alpha-M H C$ Cre(+): $A_{S K}^{+/+}$(Raf CKO:ASK ${ }^{+/+}$) and Raf-1/ASK1 double-knockout, $c$-raf-1 flox/flox: $\alpha-M H C C r e(+):$ ASK $^{-1-}$ (Raf CKO: ASK $^{-1-}$ ) mice at 10 weeks of age. (B) Western blot analysis of Raf-1 in the hearts of CTLs, Raf $\mathrm{CKO}: \mathrm{ASK}^{+/+}$, and Raf CKO:ASK-/- mice. (C and D) Echocardiographical analysis of hearts at 10 weeks of age. (C) Transthoracic M-mode echocardiographic tracings. (D) FS and diastolic left ventricle internal dimension (LVDd) in Raf CKO:ASK+/+ mice $(n=8)$ and Raf CKO:ASK-/mice $(n=7)$. The parameters in c-raf-1 flox/flox: $\alpha-M H C C r e(-): A S K^{+/+}$ $\left(\mathrm{CTL}: A S K^{+/+}\right)$mice $(n=6)$ and c-raf-1 flox/flox: $\alpha-M H C C r e(-): A S K^{-/-}$ (CTL:ASK $\left.{ }^{-1-}\right)$ mice $(n=6)$ are also shown as reference. ${ }^{*} P<0.05$ versus each group. (E) Cardiac histological analysis of the Raf-1/ ASK1 double-knockout mice. Microscopic H\&E-stained and Masson trichrome-stained sections of hearts from Raf $\mathrm{CKO}: \mathrm{ASK}^{+/+}$and Raf CKO:ASK $K^{-1-}$ mice. $(\mathbf{F})$ The phosphorylation level of JNK or p38 was assessed by immunoblotting with anti-phosphospecific antibody in Raf CKO:ASK ${ }^{-/}$and Raf CKO:ASK ${ }^{++}$hearts at 3 and 4 weeks of age. (G) The number of TUNEL-positive cells in Raf CKO:ASK ${ }^{--}$and Raf CKO: $A S K^{+/+}$hearts at 4 and 10 weeks of age. ${ }^{*} P<0.05$ versus Raf CKO: $\mathrm{ASK}^{+/+}$at the same age. Scale bar: $10 \mathrm{~mm}(\mathbf{A}), 100 \mu \mathrm{m}(\mathrm{E})$. of the loxP-flanked chloramphenicol acetyltransferase gene (30). Crossing the $\alpha$-MHCCre mice with the reporter mice resulted in Cre-mediated recombination in the heart, but not in other tissues, as determined by X-gal staining (data not shown). The $c$-raf-1 $1^{\text {flox } / \text { flox }}$ mice were mated with $\alpha$-MHCCre mice to obtain $c$-raf- $1^{f l o x} f f l o x: \alpha-M H C C r e(+)$ mice, which harbor cardiac muscle-specific Raf-1 knockout. The $A S K^{-/}$mice were in the C57BL/6J

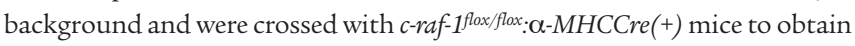
double-knockout mice [c-raf-1flox/flox: $\left.\alpha-M H C C r e(+): A^{-/-}\right]$.

This study was carried out under the supervision of the Animal Research Committee of Osaka University and in accordance with the Guidelines for Animal Experiments of Osaka University and the Japanese Animal Protection and Management Law (No. 25).

Echocardiography and hemodynamic analysis. Sequential murine transthoracic echocardiography (SONOS 5500, equipped with a $15-\mathrm{MHz}$ linear transducer; Philips Medical Systems) was performed on anesthetized mice intraperitoneally injected at 5,10 , and 15 weeks with $2.5 \%$ avertin $(8 \mu \mathrm{l} / \mathrm{g})$, as previously described (31). Hemodynamic measurements were performed on mice intraperitoneally anesthetized with $100 \mathrm{mg} / \mathrm{kg}$ ketamine and 5 $\mathrm{mg} / \mathrm{kg}$ xylazine, as done previously (32).

Histological analysis. The heart samples were arrested in diastole and immediately fixed with buffered $3.7 \%$ formalin, embedded in paraffin, and sectioned at $3 \mu \mathrm{m}$ thickness. H\&E or Masson trichrome staining was performed on serial sections.

\section{A}

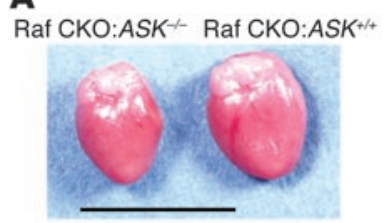

B
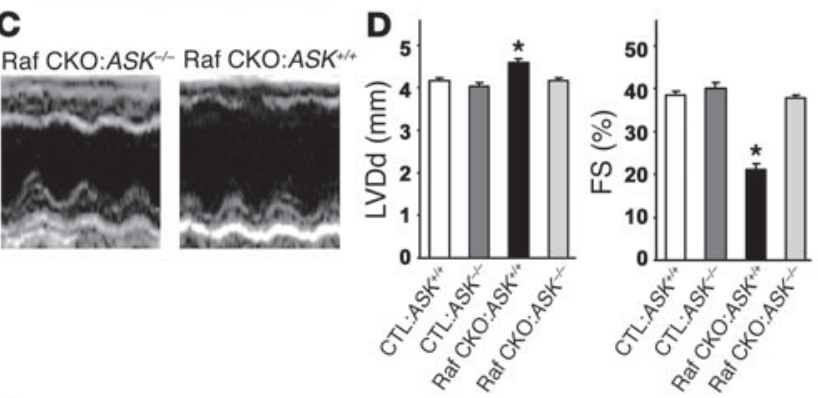

E

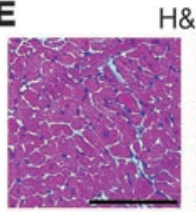

$H \& E$

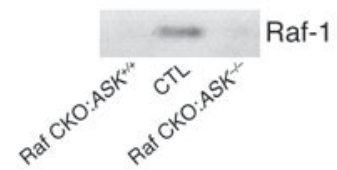

$F^{\text {Rat }}$
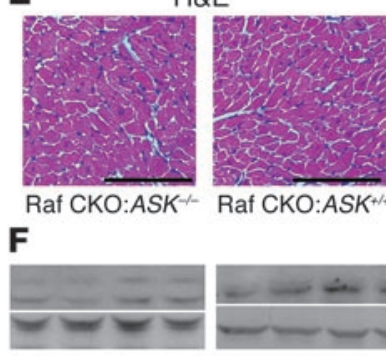

Raf CKO:ASK+/+
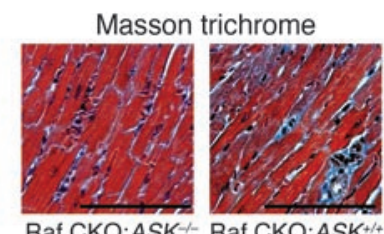

Raf $\mathrm{CKO}: \mathrm{ASK}^{-1}$ Raf $\mathrm{CKO}: \mathrm{ASK}^{+/+}$

G
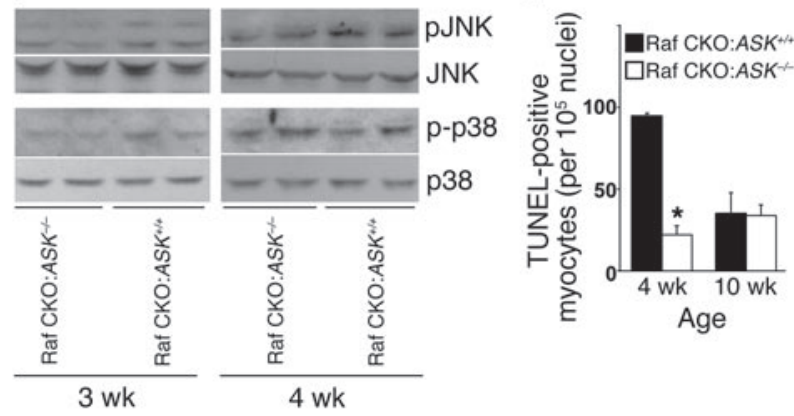
Evaluation of apoptosis. To determine the quantity of cells undergoing nuclear fragmentation, TUNEL assay was performed on paraffin-embedded heart sections using an in situ apoptosis detection kit (Takara Bio Inc.). The number of TUNEL-positive nuclei was counted by examination of the entire section with $\mathrm{a} \times 40$ objective. Triple staining with propidium iodide (Vector Laboratories Inc.), TUNEL, and anti- $\alpha$-sarcomeric actin antibody (Sigma-Aldrich) was performed.

Western blots. Mouse cardiac homogenates were prepared as previously described (33). Total protein homogenates (50 $\mu \mathrm{g} / \mathrm{lane}$ ) were subjected to Western blot analysis using antibodies against mouse Bax $(\mathrm{N}-20)$, p38 (N-20), ERK1 (K-23), JNK1 (FL), and H-Ras (C-20) from Santa Cruz Biotechnology Inc.; antibodies against MEK1/2, phospho-MEK1/2, phospho-ERK, phospho-JNK, phospho-p38, Akt, and phospho-Akt from Cell Signaling Technology Inc.; and antibody against Raf-1 from Transduction Laboratories. Western blots were developed with the ECL kit, ECL Plus kit, or ECL Advance kit (Amersham Biosciences Corp.). Quantification of signals was performed by densitometry of scanned autoradiographs with the aid of Scion Image software (version 4.02; Scion Corp.). In some studies, mice were treated before excision with endothelin-1 (50 mg/kg/min, intra-arterial perfusion) for 3 minutes to activate MAPK.

In vitro kinase assay. The activity of ASK1 was measured by immune complex kinase assay as described previously (8). Immunoprecipitation of endogenous ASK1 was performed on $500 \mu \mathrm{g}$ of myocardial extracts as reported previously (23). Immune complex kinase activity was measured as previously described (34).

Statistical analysis. Results are shown as mean \pm SEM. Paired data were evaluated by Student's $t$ test. A 1-way ANOVA with the Bonferroni's post hoc test or repeated-measures ANOVA was used for multiple comparisons. A value of $P$ less than 0.05 was considered statistically significant.

\section{Acknowledgments}

We are grateful to Ritsuko Okamoto for her expert technical assistance. This work was supported by a Grant-in-Aid for Scientific Research from the Ministry of Education, Culture, Sports, Science and Technology of Japan to K. Otsu (13470145).

Received for publication October 16, 2003, and accepted in revised form August 3, 2004.

Address correspondence to: Kinya Otsu, Department of Internal Medicine and Therapeutics, Osaka University Graduate School of Medicine, 2-2 Yamadaoka, Suita, Osaka 565-0871, Japan. Phone: 81-6-6879-3635; Fax: 81-6-6879-3645; E-mail: kotsu@medone.med.osaka-u.ac.jp.

Osamu Yamaguchi and Tetsuya Watanabe contributed equally to this work.
1. Haunstetter, A., and Izumo, S. 1998. Apoptosis: basic mechanisms and implications for cardiovascular disease. Circ. Res. 82:1111-1129.

2. Xia, Z., Dickens, M., Raingeaud, J., Davis, R.J., and Greenberg, M.E. 1995. Opposing effects of ERK and JNK-p38 MAP kinases on apoptosis. Science. 270:1326-1331.

3. Le Gall, M., et al. 2000. The p42/p44 MAP kinase pathway prevents apoptosis induced by anchorage and serum removal. Mol. Biol. Cell. 11:1103-1112.

4. Mikula, M., et al. 2001. Embryonic lethality and fetal liver apoptosis in mice lacking the c-raf-1 gene. EMBO J. 20:1952-1962.

5. Huser, M., et al. 2001. MEK kinase activity is not necessary for Raf-1 function. EMBO J. 20:1940-1951.

6. Jesenberger, V., et al. 2001. Protective role of raf- 1 in salmonella-induced macrophage apoptosis. J. Exp. Med. 193:353-364.

7. Chen, J., Fujii, K., Zhang, L., Roberts, T., and Fu, H. 2001. Raf-1 promotes cell survival by antagonizing apoptosis signal-regulating kinase 1 through a MEK-ERK independent mechanism. Proc. Natl. Acad. Sci. U. S. A. 98:7783-7788.

8. Ichijo, H., et al. 1997. Induction of apoptosis by ASK1, a mammalian MAPKKK that activates SAPK/JNK and p38 signaling pathways. Science. 275:90-94.

9. Tobiume, K., et al. 2001. ASK1 is required for sustained activations of JNK/p38 MAP kinases and apoptosis. EMBO Rep. 2:222-228.

10. Gillespie-Brown, J., Fuller, S.J., Bogoyevitch, M.A., Cowley, S., and Sugden, P.H. 1995. The mitogenactivated protein kinase kinase MEK1 stimulates a pattern of gene expression typical of the hypertrophic phenotype in rat ventricular cardiomyocytes. J. Biol. Chem. 270:28092-28096.

11. Glennon, P.E., et al. 1996. Depletion of mitogenactivated protein kinase using an antisense oligodeoxynucleotide approach downregulates the phenylephrine-induced hypertrophic response in rat cardiac myocytes. Circ. Res. 78:954-961.

12. Clerk, A., Michael, A., and Sugden, P.H. 1998 Stimulation of the $\mathrm{p} 38$ mitogen-activated protein kinase pathway in neonatal rat ventricular myocytes by the $\mathrm{G}$ protein-coupled receptor agonists, endothelin-1 and phenylephrine: a role in cardiac myocyte hypertrophy? J. Cell Biol. 142:523-535.

13. Thorburn, J., Frost, J.A., and Thorburn, A. 1994 Mitogen-activated protein kinases mediate changes in gene expression, but not cytoskeletal organization associated with cardiac muscle cell hypertrophy. J. Cell Biol. 126:1565-1572.

14. Post, G.R., Goldstein, D., Thuerauf, D.J., Glembotski, C.C., and Brown, J.H. 1996. Dissociation of p44 and p42 mitogen-activated protein kinase activation from receptor-induced hypertrophy in neonatal rat ventricular myocytes. J. Biol. Chem. 271:8452-8457.

15. Ramirez, M.T., et al. 1997. The MEKK-JNK pathway is stimulated by alpha1-adrenergic receptor and ras activation and is associated with in vitro and in vivo cardiac hypertrophy. J. Biol. Chem. 272:14057-14061.

16. Bueno, O.F., et al. 2000. The MEK1-ERK1/2 signaling pathway promotes compensated cardiac hypertrophy in transgenic mice. EMBO J. 19:6341-6350.

17. Agah, R., et al. 1997. Gene recombination in postmitotic cells: targeted expression of cre recombinase provokes cardiac-restricted, site-specific rearrangement in adult ventricular muscle in vivo. J. Clin. Invest. 100:169-179.

18. Aikawa, R., et al. 1997. Oxidative stress activates extracellular signal-regulated kinases through src and ras in cultured cardiac myocytes of neonatal rats. J. Clin. Invest. 100:1813-1821.

19. Oltvai, Z.N., Milliman, C.L., and Korsmeyer, S.J. 1993. Bcl-2 heterodimerizes in vivo with a conserved homolog, Bax, that accelerates programmed cell death. Cell. 74:609-619.

20. Kanamoto, T., et al. 2000. Role of apoptosis signal-regulating kinase in regulation of the c-Jun $\mathrm{N}$-terminal kinase pathway and apoptosis in sympathetic neurons. Mol. Cell. Biol. 20:196-204.

21. Yamaguchi, O., et al. 2003. Targeted deletion of apoptosis signal-regulating kinase 1 attenuates left ventricular remodeling. Proc. Natl. Acad. Sci. U. S. A. 100:15883-15888,

22. Chen, J., Kubalak, S., and Chien, K. 1998. Ventricular muscle-restricted targeting of the RXRalpha gene reveals a non-cell-autonomous requirement in cardiac chamber morphogenesis. Development.
125:1943-1949.

23. Saitoh, M., et al. 1998. Mammalian thioredoxin is a direct inhibitor of apoptosis signal-regulating kinase (ASK) 1. EMBO J. 17:2596-2606.

24. Kuan, C., et al. 1999. The Jnk1 and Jnk2 protein kinases are required for regional specific apoptosis during early brain development. Neuron. 22:667-676.

25. Hatai, T., et al. 2000. Execution of apoptosis signalregulating kinase 1 (ASK1)-induced apoptosis by the mitochondria-dependent caspase activation. J. Biol. Chem. 275:26576-26581.

26. Chang, H.Y., Nishitoh, H., Yang, X., Ichijo, H., and Baltimore, D. 1998. Activation of apoptosis signalregulating kinase 1 (ASK1) by the adapter protein Daxx. Science. 281:1860-1863.

27. Zimmermann, S., and Moelling, K. 1999. Phosphorylation and regulation of raf by Akt (protein kinase B). Science. 286:1741-1744.

28. Kim, A.H., Khursigara, G., Sun, X., Franke, T.F., and Chao, M.V. 2001. Akt phosphorylates and negatively regulates apoptosis signal-regulating kinase 1. Mol. Cell. Biol. 21:893-901.

29. Du, J., Cai, S.-H., Shi, Z., and Nagase, F. 2004. Binding activity of $\mathrm{H}$-Ras is necessary for in vivo inhibition of ASK1 activity. Cell Res. 14:148-154.

30. Sakai, K., and Miyazaki,J. 1997. A transgenic mouse line that retains Cre recombinase activity in mature oocytes irrespective of the cre transgene transmission. Biochem. Biophys. Res. Commun. 237:318-324.

31. Tanaka, N., et al. 1996. Transthoracic echocardiography in models of cardiac disease in the mouse. Circulation. 94:1109-1117.

32. Nakayama, H., et al. 2002. Cardiac-specific overexpression of a high $\mathrm{Ca}^{2+}$ affinity mutant of SERCA2a attenuates in vivo pressure overload cardiac hypertrophy. FASEB J. doi:10.1096/fj.02-0474fje.

33. Weinbrenner, C., Liu, G.-S., Cohen, M., and Downey, J. 1997. Phosphorylation of tyrosine 182 of p38 mitogen-activated protein kinase correlates with the protection of preconditioning in the rabbit heart. J. Mol. Cell. Cardiol. 29:2383-2391.

34. Hirotani, S., et al. 2002. Involvement of nuclear factor- $\mathrm{KB}$ and apoptosis signal-regulating kinase 1 in G-protein-coupled receptor agonist-induced cardiomyocyte hypertrophy. Circulation. 105:509-515. 